\title{
Portada / Revisores / Indice
}

FAVE Sección Ciencias Agrarias es la revista oficial de la Facultad de Ciencias Agrarias de Esperanza (Universidad Nacional del Litoral) y tiene por objetivo publicar trabajos originales e inéditos, pudiendo ser artículos científicos, comunicaciones, revisiones y notas, brindando un medio que permita a los investigadores nacionales e internacionales publicar sus resultados, contribuyendo al desarrollo científicotecnológico en el campo disciplinar de las Ciencias Agrarias y Forestales.

La revista es de frecuencia semestral, publicándose un volumen por año con dos números, en junio (Enero-Junio) y diciembre (Julio-Diciembre) de cada año. El idioma oficial de la revista es el español, aceptándose además trabajos en portugués e inglés.

FAVE - Sección Ciencias Agrarias es continuación de la Revista FAVE (ISSN 0325 3112), Publicación que fuera incorporada al Núcleo Básico de Revistas Científicas Argentinas del Consejo Nacional de Investigaciones Científicas y Técnicas (CONICET), Scielo, DOAJ e indexada en Abstracts del C.A.B. International y CATIE - IICA.; y en REDIB.

La Revista FAVE - Sección Ciencias Agrarias agradece a los siguientes revisores el arbitraje de los artículos evaluados en este período:

Dr. Rubén Omar Arias - Facultad de Ciencias Agrarias y Forestales (UNLP)

MSc. Verónica Charlon - INTA Estación Experimental Agropecuaria Rafaela, Santa Fe Dra. Fabiana Gallardo - Facultad de Ciencias Naturales y Museo (UNLP)

Dra. Isabel María Lacau-Mengido - IBYME - CONICET

Dr. Juan Carlos Martínez González - Universidad Autónoma de Tamaulipas, México PhD. Alejandro Mendoza - INIA La Estanzuela, Uruguay

Ing. Agr. Carlos Mirábile - Instituto Nacional del Agua, Mendoza

Dra. Cecilia Panigatti - Facultad Regional Rafaela (UTN)

Ing. Agr. Ernesto Schilder - INTA - Estación Experimental Agropecuaria Rafaela, Santa Fe MSc. Oscar Siliquini - Facultad de Agronomía (UNLPam)

Dr. Jorge Tizado - Universidad de León. Ponferrada, España

Dra. Alicia Florencia Urteaga Omar - Facultad de Ciencias Agropecuarias (UNER)

\section{INDICE}

Volumen 18, $\mathrm{N}^{0} 1,2019$

Permeado de suero como abono: Respuesta de cultivos y cambios en un natrustol.

BADINO, O.N., FELLI, O.; PILATTI, M. A.; GHIBERTO, P. J. \& MIRETTI, M.C.

Relationship between damage on leaves caused by Liriomyza huidobrensis (Diptera, Agromizydae), adults trapped in sticky traps and parasitoids in a commercial crops of Cucurbita maxima var. zapallito (Cucurbitaceae).

BERTOLACCINI, I.; CURIS, M.C.; LUTZ, A.; MARTINEZ, J.J.; FAVARO, J.C. \&

TROD, A. 
Estimación de costos de inversión para un estudio de caso: dren topo y dren con tubería en la Región Central Santafesina.

CAMUSSI, G.; MAINA, M.; TRAVADELO, M. \& MARANO, R.

Efecto de diferentes mulchings sobre el crecimiento de una plantación de durazneros (Prunus persicae L.) variedad Opodepe en Zavalla, Provincia de Santa Fe.

FLORES, P.; AUSILIO, A.; LEONE, A.; POGGI, D.; QUADRELLI, A.; SOLINI, I.; LÓPEZ, M.; MONTI, F. \& CATRARO, M.

Efectos de la inclusión de taninos condensados de Schinopsis balansae en la dieta de cabras en lactancia sobre parámetros productivos.

MARTÍNEZ, G.M.; SUÁREZ, V.H.; OLMOS, L.H.; ALFARO, R.J.; ALFARO, E.J.; YANES, R.P. \& OROSCO, S.M.

Comportamiento ingestivo de agua en vacas lecheras (Datos preliminares).

TOFFOLI, G.D.; LEVA, P.E.; GHIANO, J.; MILLAPÁN, L.; COSTAMAGNA, D.; TAVERNA, M.; WALTER, E.; CORONEL, V. \& NUÑEZ. S. 